\title{
Dynamic aerofracture of dense granular packings
}

\author{
Michael J. Niebling, ${ }^{1,2}$ Renaud Toussaint, ${ }^{2,3}$ Eirik G. Flekkøy, ${ }^{1,3}$ and Knut Jørgen Måløy ${ }^{1,3}$ \\ ${ }^{1}$ Department of Physics, University of Oslo, P.O. Box 1048, 0316 Oslo, Norway \\ ${ }^{2}$ Institut de Physique du Globe de Strasbourg, Ecole et Observatoire des Sciences de la Terre, Université de Strasbourg, \\ CNRS, 5 rue Descartes, F-67000 Strasbourg, France \\ ${ }^{3}$ Centre for Advanced Study at the Norwegian Academy of Science and Letters, Drammensveien 78, 0271 Oslo, Norway
}

(Received 12 October 2011; revised manuscript received 28 September 2012; published 26 December 2012)

\begin{abstract}
A transition in hydraulically induced granular displacement patterns is studied by means of discrete numerical molecular dynamics simulations. During this transition the patterns change from fractures and fingers to finely dispersed bubbles. The dynamics of the displacement patterns are studied in a rectangular Hele-Shaw cell filled with a dense but permeable two-dimensional granular layer. At one side of the cell the pressure of the compressible interstitial gas is increased. At the opposite side from the inlet of the cell a semipermeable boundary is located. This boundary is only permeable towards the gas phase while preventing grains from leaving the cell. The imposed pressure gradient compacts the grains. In the process we can identify and describe a mechanism that controls the transition of the emerging displacement patterns from fractures and fingers to finely dispersed bubbles as a function of the interstitial gas's properties and the characteristics of the granular phase.
\end{abstract}

DOI: 10.1103/PhysRevE.86.061315

PACS number(s): 81.05.Rm, 47.54.-r, 62.25.Mn

\section{INTRODUCTION}

Hydraulic fractures occur when diverse materials break under the stress induced by fluids or gases. Understanding how hydraulic fracturing is initiated and progresses is of fundamental importance whenever safe dams are constructed, supercritical $\mathrm{CO}_{2}$ is stored, or sustainable wells are drilled. Volcanic dikes and sills arise naturally by hydraulic fracturing processes. Just as we learn to protect ourselves from the unwanted effects of hydraulic fractures, it has been proven to be a useful technology to fracture the reservoir rock formations around a well bore to enhance the recovery of mineral oil and natural gas.

In previous studies [1-17] experiments have been conducted in circular and rectangular Hele-Shaw cells filled with small grains. Under air injection in the center of the circular cell, multiple fingers of low particle density emerged from the inlet $[1,2]$.

For the rectangular cell it was observed that a decompaction front travels first from the outlet to the inlet before a few fingers of low particle density grow from the air inlet $[3,4]$. Furthermore, in a recent work, the coarsening of such fingers was studied [5]. In Ref. [6] loosely compacted grains were exposed to a pressure gradient. In the process of these simulations and experiments a spinodal-like instability was observed that displayed emerging and growing dispersed bubbles of low particle density.

Considering this background various dynamics and structures have been observed and described. However, a theory accounting for the transition from dynamics that display dispersed bubbles to dynamics that display fractures and fingerlike patterns has not been presented. In this article we will study and explain the mechanism that controls the transition between these two types of emerging structures during the compaction of a granular layer.

After a number of experiments have been performed in a rectangular cell [18], we chose to perform a numerical simulation. Using a discrete numerical molecular dynamic model, we have thereby the possibility to systematically and independently vary the viscosity of the interstitial fluid. Effectively this corresponds to changing the size of the system, as we will discuss in the following. This approach allows us to study system sizes that experimentally would be extremely complicated and dangerous to achieve. In the following section we will briefly discuss the parameters and the setup used for the numerical model.

\section{SETUP OF THE NUMERICAL MODEL}

The setup is illustrated in Fig. 1. Two glass plates are separated by $1 \mathrm{~mm}$. The space in between the glass plates is filled with dense grains. The gas phase injected at the inlet following the increase of pressure is identical to the gas saturating initially the porous packing. The solid volume fraction of the grains is $\rho_{s}^{(0)}=0.42$, which is less than the maximum of $\rho_{s}^{(\max )}=0.60$ to allow compaction of the grains. The pressure at the inlet is set to a constant value of $P_{I}=2.5 \times 10^{5} \mathrm{~Pa}$. The outlet is located on the opposite side of the cell. Here a semipermeable boundary stops the grains from leaving the cell but is open towards the gas phase. Apart from the semipermeable boundary at the outlet, all three other boundaries are fully sealed. There gas or particle exchange is impossible. Around 200000 grains of $140 \pm 10 \% \mu \mathrm{m}$ in diameter are simulated. The size distribution of $10 \%$ is artificially set to suppress the formation of a triangular grain packing. Initially the particles are inserted into the cell, each with a random velocity. Shortly after the friction between particles and the plates and energy dissipation during particles collisions is activated and the particles build up a fixed random packing after they lost their kinetic energy. At the start of the simulations the pressure at the inlet is increased; just after the pressure was increased a pressure front starts to propagate through the cell and compacts the particles. The length of the pressure front skin depth is adjusted and varied by changing the gas viscosity. 


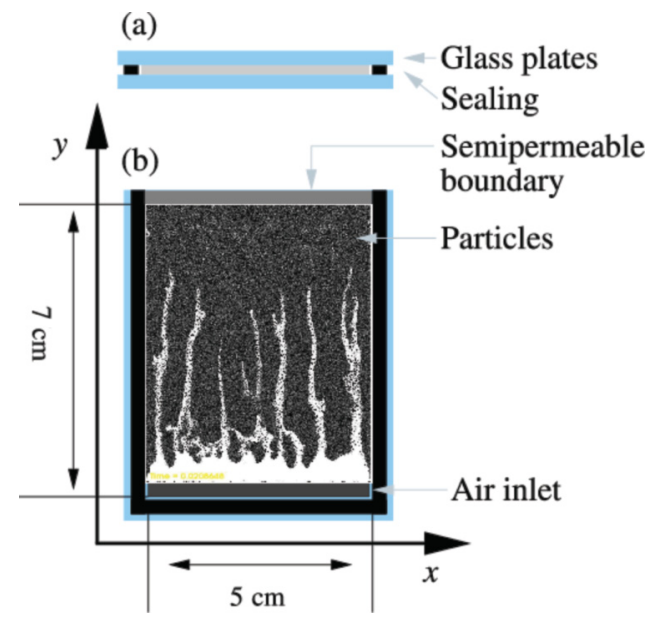

FIG. 1. (Color online) Setup of the system: (a) cross section and (b) top view. Compressed air is injected at the inlet. The outlet is semipermeable: open towards the gas phase and closed for the granular phase.

\section{THEORY AND SIMULATIONS}

The numerical model employed is a two-dimensional hybrid model. It uses a continuum description for the gas phase and a discrete description of the granular phase. Friction between particles and the top and bottom plates is included. It was compared to well matched experiments and shown to reproduce closely the dynamics of granular flows at low Reynolds numbers [19-21] and under conditions similar to those assumed in the present article [1].

\section{DYNAMICS OF THE GAS PHASE:}

The equations ruling the evolution of the pressure $P=$ $\tilde{P}+P_{0}$, where $P_{0}$ is the atmospheric pressure and $\tilde{P}$ is the local pressure fluctuation, are derived in detail in Refs. [1,19, 20,22-26]. We calculate $P$ according to

$$
\phi\left[\frac{\partial P}{\partial t}+\mathbf{u} \cdot \nabla P\right]=\nabla \cdot\left[P \frac{\kappa}{\mu_{f}} \nabla P\right]-P \nabla \cdot \mathbf{u} .
$$

This equation basically describes how the divergence of the local granular velocity u leads to local pressure changes according to the gas displacement by the grains and between them. The viscosity $\mu_{f}$ of the gas determines how fast the gas diffuses through the permeable grains to equalize the pressure fluctuations. The local porosity is $\phi=1-\rho_{s}$ and the local permeability $\kappa$ is calculated by the Carman-Kozeny relation $[22,27]$. Equation (1) is derived from mass conservation of the gas, mass conservation of the granular medium, and by assuming a local Darcy law.

\section{DYNAMICS OF THE PARTICLES}

The dynamics of each individual particle with the velocity $v_{p}$, particle mass $m=\rho_{m} \pi a^{2} h$, particle mass density $\rho_{m}$, volume $V_{a}=\pi a^{2} h$ in a cell with a plate spacing of $h$, and the number density defined as $\rho_{n}=\rho_{s} \rho_{m} / m$ is described by

$$
m \frac{d v_{p}}{d t}=\mathbf{F}_{\mathbf{I}}+\mathbf{F}_{\mathbf{d}}+\mathbf{F}_{\mathbf{a}}-\frac{\nabla P}{\rho_{n}},
$$

where $\mathbf{F}_{\mathbf{I}}$ is the linear interparticle solid contact force. The third term on the right-hand side of Eq. (2) arises from the momentum exchange between the gas and granular phase. Here $\mathbf{F}_{\mathbf{d}}$ is a viscous force accounting for energy dissipation during particle collisions. More details are given in Refs. [19,20].

In the granular packing we assume that the normal stress $P_{g}^{\perp}$ is proportional to the in-plane stress $P_{g}^{\|}$by a factor $\lambda$. This relation is known as the Janssen hypothesis $[1,19]$. Considering also a Coulomb friction model, we get that the frictional force $F_{a}$ per particle with the glass plates is proportional to the normal stress by a friction coefficient $\gamma$ :

$$
F_{a} \leqslant \gamma S_{a}\left(2 P_{g}^{\perp}+\rho_{m} g h\right)=\gamma S_{a}\left(2 \lambda P_{g}^{\|}+\rho_{m} g h\right) .
$$

The factor 2 in the first term accounts for the two glass plates on each side of the particle. Here $S_{a}=\pi a^{2}$ is the contact area of the particles with the plates. The second term is a contribution due to the gravitational acceleration $g$ on the grains, which leads to additional friction between the bottom plate and the particles. This term is included for completeness, but has a negligible effect on the following results. Finally, the particle propagation is modeled by the velocity Verlet scheme [28,29].

\section{RESULTS}

The coefficients that determine the friction with the glass plates are set to $\gamma \lambda=4.0$, a rather high value. Lower values have also been tested and resulted in less branched fractures. The pressure at the inlet is increased as a steep ramp, fast enough that the maximum pressure at the inlet of $P_{I}=$ $2.5 \times 10^{5} \mathrm{~Pa}$ is reached before particles significantly start to move. The injected gas is considered as an ideal gas and has the compressibility of air $\beta_{T}=1 / P_{0}$. The compressibility is kept constant. The gas viscosity, however, is increased gradually in different simulations, from the value for air $\mu_{f}^{(\text {air) }}=$ $0.018 \mathrm{mPas}$ by a factor of 1000 up to $\mu_{f}=18.0 \mathrm{mPas}$. The results of the simulations for the particle density are shown in Fig. 2 (see also Ref. [30]). Dark regions correspond to a high particle density while brighter areas represent low particle density. The gas viscosity decreases from top to bottom while time progresses from left to right. In the time sequences the emerging structures change drastically as a function of the gas viscosity. In the simulation with the lowest gas viscosity dispersed bubbles of reduced particle density appear in the whole cell apart from a darker region at the outlet, where particles get compacted at the semipermeable boundary [see Fig. 2 for $\mu_{f}=0.18-0.018 \mathrm{mPas}$ and $t=0.004-0.01 \mathrm{~s}$ or Fig. 3(b) for a close-up]. Increasing the viscosity, structures change from dispersed bubbles to fractures. Furthermore, compaction of the grains occurs here at the inlet and in front of the fingers instead of at the outlet. Taking a close look at the simulations at high viscosity in Fig. 2, this is indicated by a dark region in front of the fractures and fingers that was absent for low viscosity gas [see also Fig. 3(a) for a close-up].

For a better quantification, we represent in Fig. 4 the dependence of three quantities as a function of $y$ across the cell, at different times. The choice of parameters represented allows us to understand whether grains close to the boundary are pushed through solid stress or fluid drag: The solid volume 


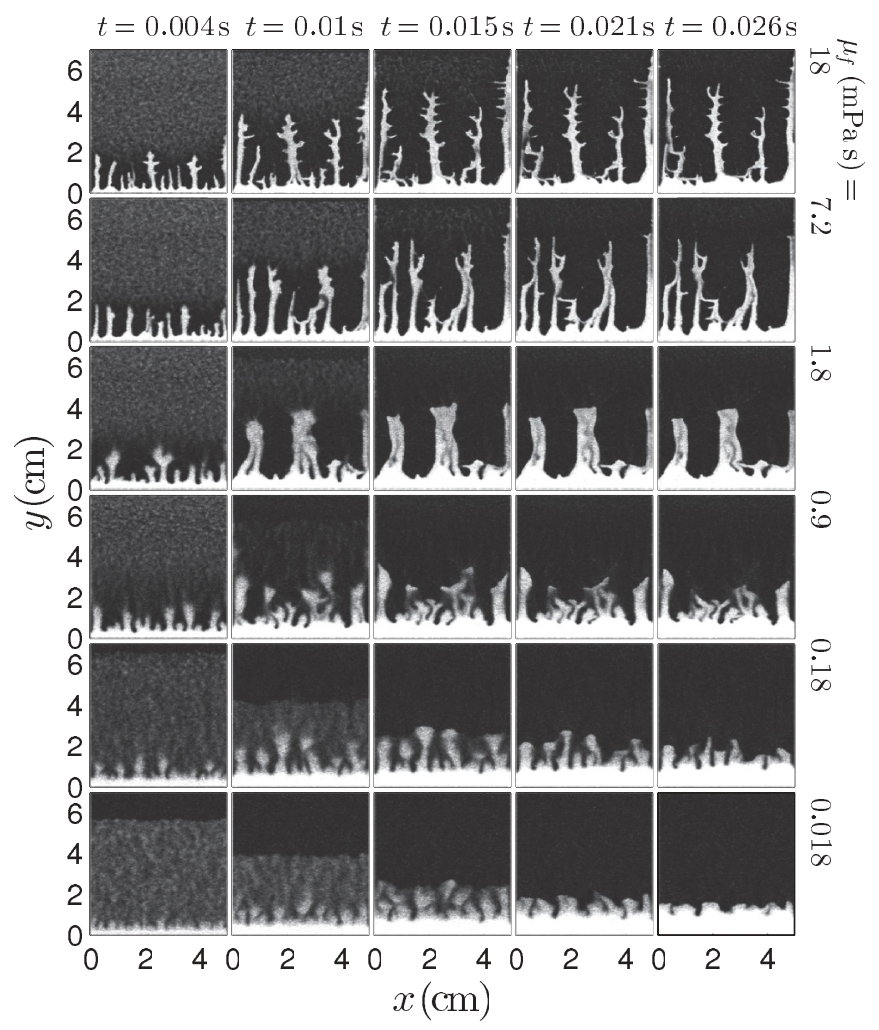

FIG. 2. Snapshots during the simulations of the particle density in the Hele-Shaw cell, displayed for decreasing gas viscosity from top to bottom and for increasing time (left to right). Low particle density appears brighter in the snapshots. Under air injection, fractures, fingers, and dispersed bubbles of low particle density emerge and propagate in time towards the outlet of the cell. The $y$ axis specifies the distance from the air inlet in $\mathrm{cm}$. The maximal density is normalized to one.

fraction corresponds to the grain density and allows us to clarify the formation of compacted zone in different regions, which play an important role in the system dynamics. The grain velocity allows us to image where and when the packing deforms, and the pressure gradient is directly related to the fluid drag force.

The solid volume fraction is averaged along the $x$ direction and plotted as a function of the $y$ direction for three time steps in Figs. 4(a)-4(c). In these figures zones of compacted grains correspond to solid volume fraction values larger than 0.45 . The inlet side of the cell is just like before located at a $y$ position close to zero. The outlet is located at $y=6.9 \mathrm{~cm}$. At the first time step in Fig. 4(a) we notice that compaction fronts arise at the inlet side for viscosity values of $\mu_{f}<0.018 \mathrm{mPa} \mathrm{s}$ and at the outlet side of the cell for the lowest gas viscosity of $\mu_{f}=0.018 \mathrm{mPa}$ s. During the next time steps in Figs. 4(b) and 4(c) the compaction fronts at the inlet progress towards the outlet. At the outlet compaction of grains now also appears for simulations with gas viscosity values up to $\mu_{f}=0.9 \mathrm{mPas}$. The zone of particle compaction at the outlet grows and expands in time towards the inlet.

To understand how the grain compaction depends on the gas viscosity we will have to take the pressure evolution in the cell into account. In Fig. 5 the gas pressure in the cell is displayed for time steps and gas viscosity values corresponding to Fig. 2.

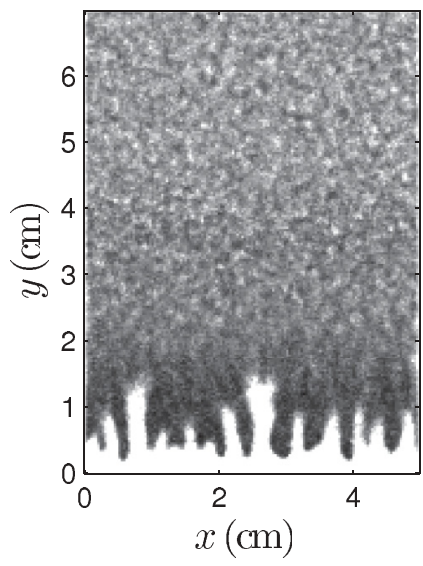

(a)

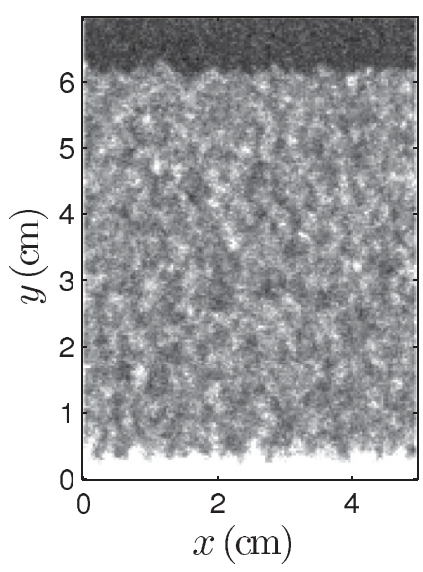

(b)
FIG. 3. Close-up of snapshots of the particle density in the Hele-Shaw cell during the simulations at $t=0.003 \mathrm{~s}$. The snapshots correspond to the plots in Figs. 4(a), 4(d), and 4(g). In 3(a) $\mu_{f}=$ $1.8 \mathrm{mPa} \mathrm{s}$ and in (b) $\mu_{f}=0.018 \mathrm{mPa}$. The density is normalized to one and the color bar range is chosen from 0 to 0.5 to enhance the density contrast. In the plots the darker areas represent higher grain density. In (a) a compaction front at the inlet around the finger tips has emerged. In (b) grains get compacted at the outlet of the cell, which corresponds to a darker stripe at the outlet (for $y>6 \mathrm{~cm}$ ).

The bottom row, where the gas viscosity has the smallest value in this figure, shows that the pressure decays continuously towards the outlet. Alternatively we can look at Fig. 4(d), where the pressure average in the $x$ direction is plotted as a function of the $y$ direction. Confirming our observation from above, Fig. 4(d) shows a linear pressure profile in the $y$ direction for the lowest viscosity right at the start of the simulation. In this regime the pressure gradient acts as a body force displacing all particles in the cell simultaneously and homogeneously and therefore preventing particles from jamming. Furthermore, the homogeneous grain motion is also confirmed for the low viscosity value in Fig. $4(\mathrm{~g})$. Here $u_{y}$, the $y$ component of the particle velocity averaged along the $x$ direction, is constant as a function of $y$.

The appearance of low particle density bubbles under these conditions has previously been reported and described in Ref. [6]. However, in the present setup the semipermeable boundary at the outlet interferes with the otherwise uniform particle motion. This results in the previously mentioned compaction layer at the outlet.

The pressure decay in Fig. 5 becomes more localized if the viscosity is increased. Now the pressure drops rapidly at the interface between the granular phase and the growing particle free region at the inlet side of the cell. Alternatively we can confirm this localized pressure decay in Figs. 4(d)-4(f), where the pressure is averaged along the $x$ direction and plotted as a function of $y$. This time sequence of plots shows that increasing the viscosity results in an increasingly steep pressure decay at the interface. Further inside the granular packing towards the outlet the pressure remains initially unchanged. In this regime the pressure gradient acts as a surface force on the particles at the interface between granular and gas phase. Due to the localized pressure gradient, particles at the interface close to the pressure inlet are initially accelerated more quickly than 


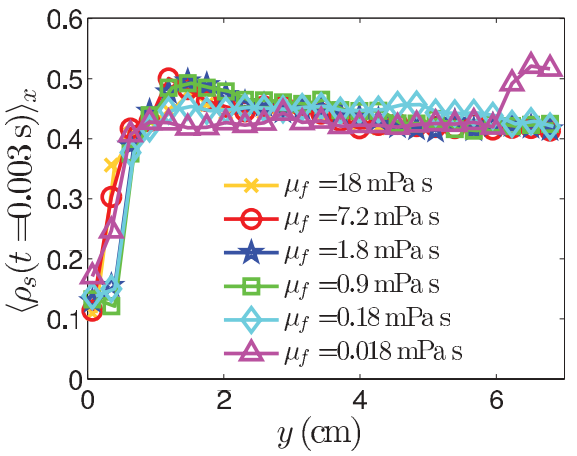

(a)

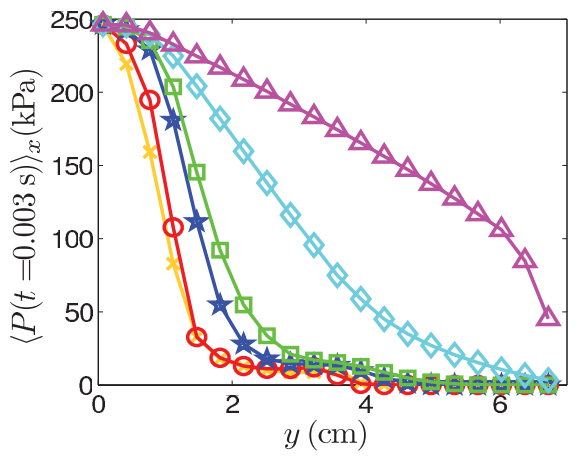

(d)

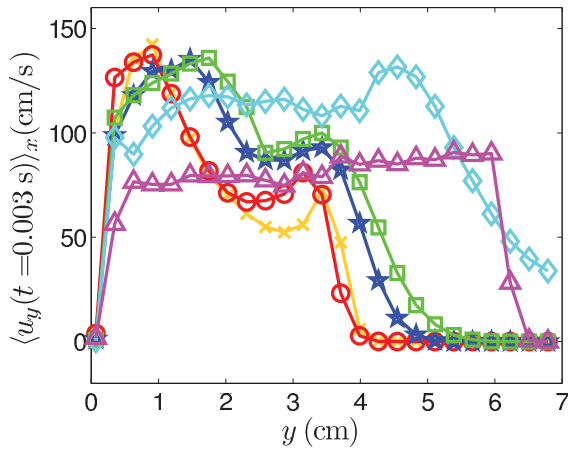

(g)

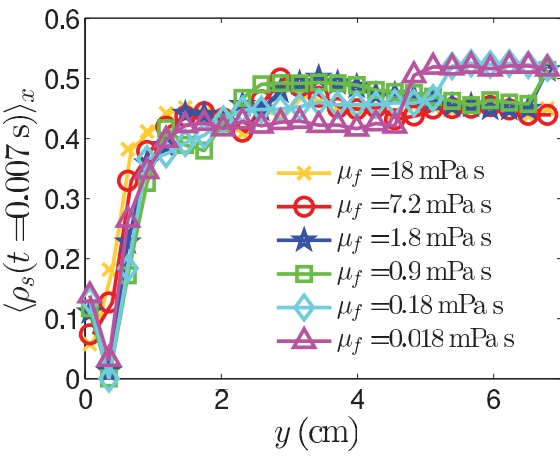

(b)

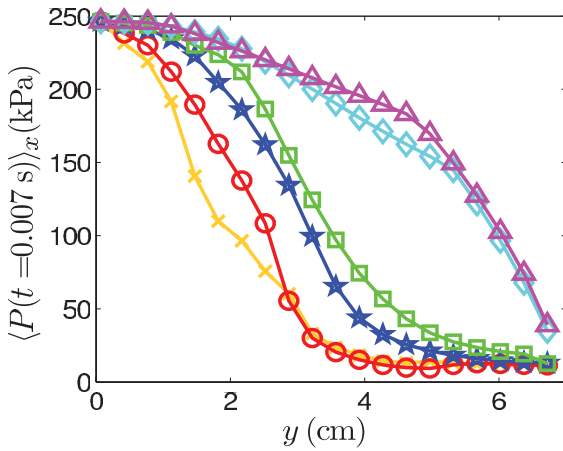

(e)

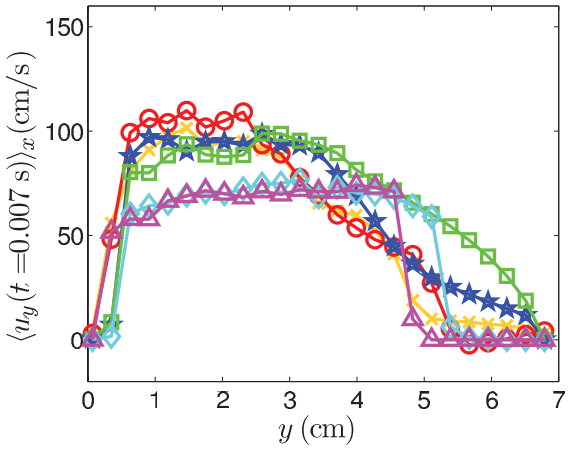

(h)

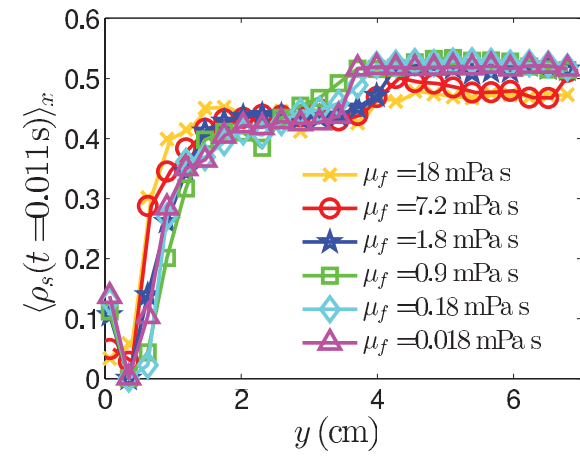

(c)

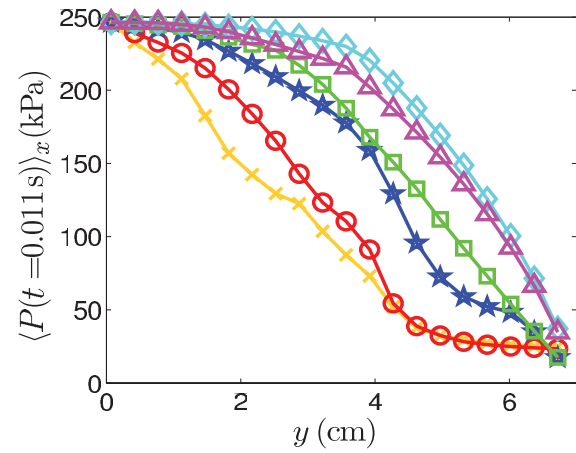

(f)

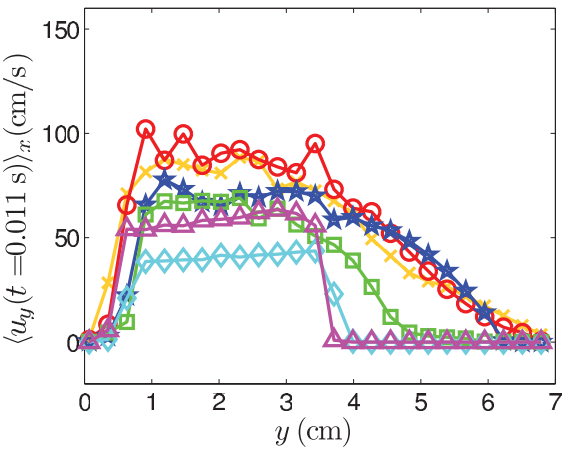

(i)

FIG. 4. (Color online) Several quantities have been averaged along the $x$ direction and are plotted in rows from top to bottom: (a)-(c) solid volume fraction $\rho_{s}$, (d)-(f) pressure $P$, and (g)-(i) $y$ component of the grain velocity $u_{y}$. From left to right the three figures in each row correspond to the times $t=0.003,0.007$, and $0.011 \mathrm{~s}$ after the start of the injection. The legend is consistent in all plots and is displayed only in the first row.

the particles close to the outlet. This leads to particle collisions and jamming in the granular packing at the interface where a compaction front builds up.

Where the grains are compacted the solid stress increases as displayed in Fig. 6. The solid stress is shown to increase first at the outlet for the least viscous gas. For higher viscosity values the solid stress is localized around the finger tips. Here the solid stress decays inside the granular packing over a certain distance, which is the same size as the compaction front. This distance can be larger than the distance over which the pressure gradient decays from the interface into the granular packing. In this regime the particles further inside the packing are accelerated through solid contacts rather than by the pressure gradient of the fluid. Such solid contacts in a compacted granular medium transmit the stress localized along distinct force chains. This causes a heterogeneous acceleration of the particles and the particle velocity in the $y$ direction is increased along certain localized paths, as shown in Fig. 7. Local noise and disturbance are now affecting the evolution of the interface directly and fractures appear.

In this situation local particle rearrangement and jamming results in the observed fracture pattern. To quantify the transition between the two regimes we will briefly define the characteristics of the pressure diffusion, as done in detail in Ref. [20]. We neglect the motion of the granular phase and consider a standard diffusion equation for the pressure evolution within the granular phase. This approach allows us to define a diffusion constant for the pressure

$$
D=\frac{\kappa}{\left(1-\rho_{s}\right) \beta_{T} \mu_{f}} .
$$




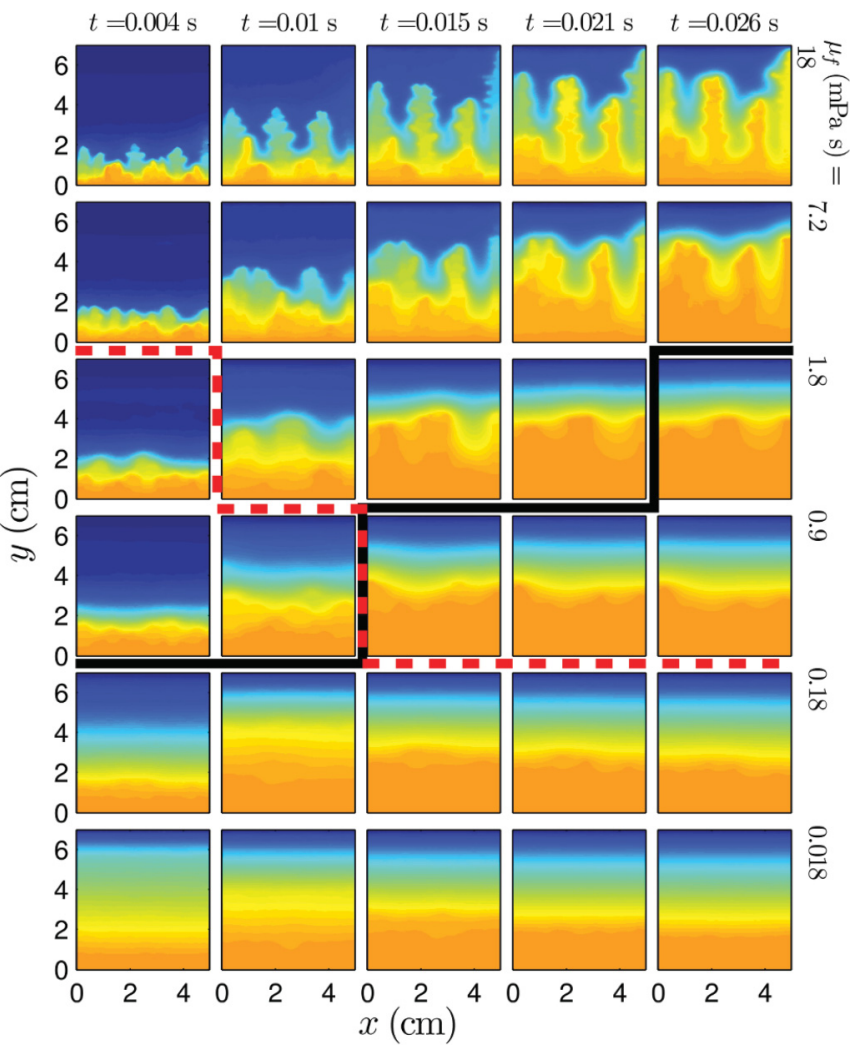

FIG. 5. (Color online) Snapshots show the pressure evolution for decreasing gas viscosity (top to bottom) and as a function of time (left to right). High pressure appears yellow (brighter) in the snapshots. The dashed (red) line between the snapshots shows the time $t_{f}$ [see Eq. (7)]. In the snapshots above this dashed (red) line the compaction front thickness is greater than the skin depth of the pressure $s$ defined in Eq. (5). Below the black line the skin depth $s$ has reached half of the system size in a theoretical system where particles do not move. The maximal pressure is normalized to one.

Furthermore, we define a skin depth

$$
s=\sqrt{4 D t},
$$

where $s$ is the distance from the gas-particle interface over which the pressure has decayed by $P(s)=\frac{1}{e} P_{I}$ [31]. The diffusion constant in Eq. (4) specifies how fast the fluid flow can equalize pressure changes at given gas properties and characteristics of the porous medium.

We can compare how well this assumed pressure evolution fits to the simulations. For this purpose we average the pressure in the $x$ direction and calculate the maximum gradient in the $y$ direction of this pressure average: $\max \left(\partial_{y}\langle P\rangle_{x}\right)$ as a function of time [see Fig. 8(b) for all viscosity values]. The $y$ position of $\max \left(\partial_{y}\langle P\rangle_{x}\right)$ propagates from the inlet towards the outlet as time passes as shown in Fig. 8(a). At low viscosity the position of the maximum gradient reaches the outlet almost immediately after the simulation has started while at high viscosity values this maximum gradient never reaches the outlet during the simulated time.

In Fig. 8(a) the $y$ position of $\max \left(\partial_{y}\langle P\rangle_{x}\right)$ depends on the combined position of the skin depth and the position of the gas-particle interface. To study the pressure evolution relative

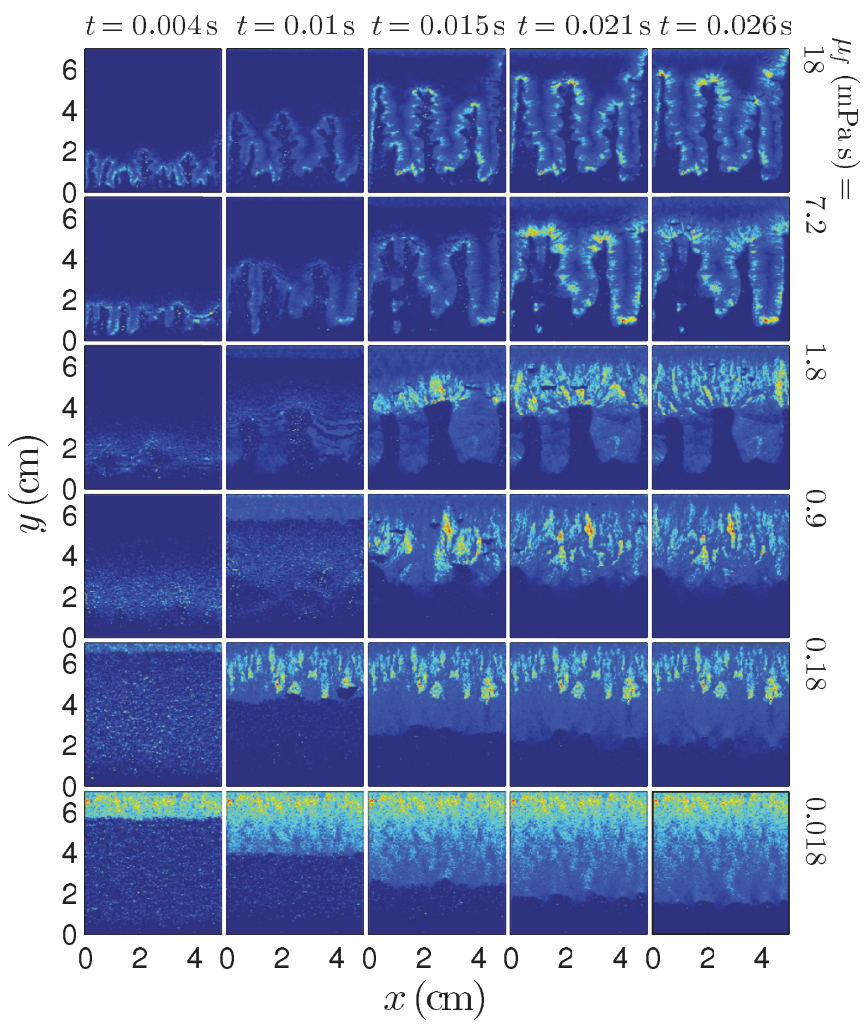

FIG. 6. (Color online) Snapshots of the normal solid in-plane stress between the particles for decreasing gas viscosity (top to bottom) and as a function of time (left to right). High stress appears yellow (brighter) in the snapshots. The maximal normal solid in-plane stress is normalized to one.

to the gas-particle interface at the finger tip in a Lagrangian reference frame it is more useful to calculate $\max \left(\partial_{y}\langle P\rangle_{x}\right)$ as a function of time. If the assumed propagation of the skin depth for the pressure as a square root in time relative to the interface is correct we should be able to rescale $\max \left(\partial_{y}\langle P\rangle_{x}\right)$ by multiplication with the skin depth $s(t)$ defined in Eq. (5). The rescaling is done in Fig. 8(c) and Fig. 9 shows a close-up of the graphs with the four highest viscosity values tested. The rescaled graphs are constant in time. This supports the proposed diffusive pressure behavior relative to the particle motion. For low viscosity values the rescaling does not result in a constant behavior because of the limiting finite size of the cell. Furthermore, we observe that the plots do not fall directly above each other. For increasing viscosity values the graphs are shifted downwards by a constant. This effect is not captured in our explanation and is most likely due to rearrangements and motion of the particles. In the following the skin depth is used as a measure of how steep and how far the pressure decays into the granular phase in the cell.

We notice in Eqs. (4) and (5) that increasing the viscosity reduces the skin depth. Using Eq. (5), the calculated time for the skin depth to propagate through the cell in the $y$ direction is $0.0009 \mathrm{~s}$ at the lowest viscosity value. This is faster than the time needed to notice a significant movement of the grains. For the highest viscosity value in the simulations the calculated time is $0.9 \mathrm{~s}$ until the skin depth has grown to the size of the cell assuming a fixed granular packing. 


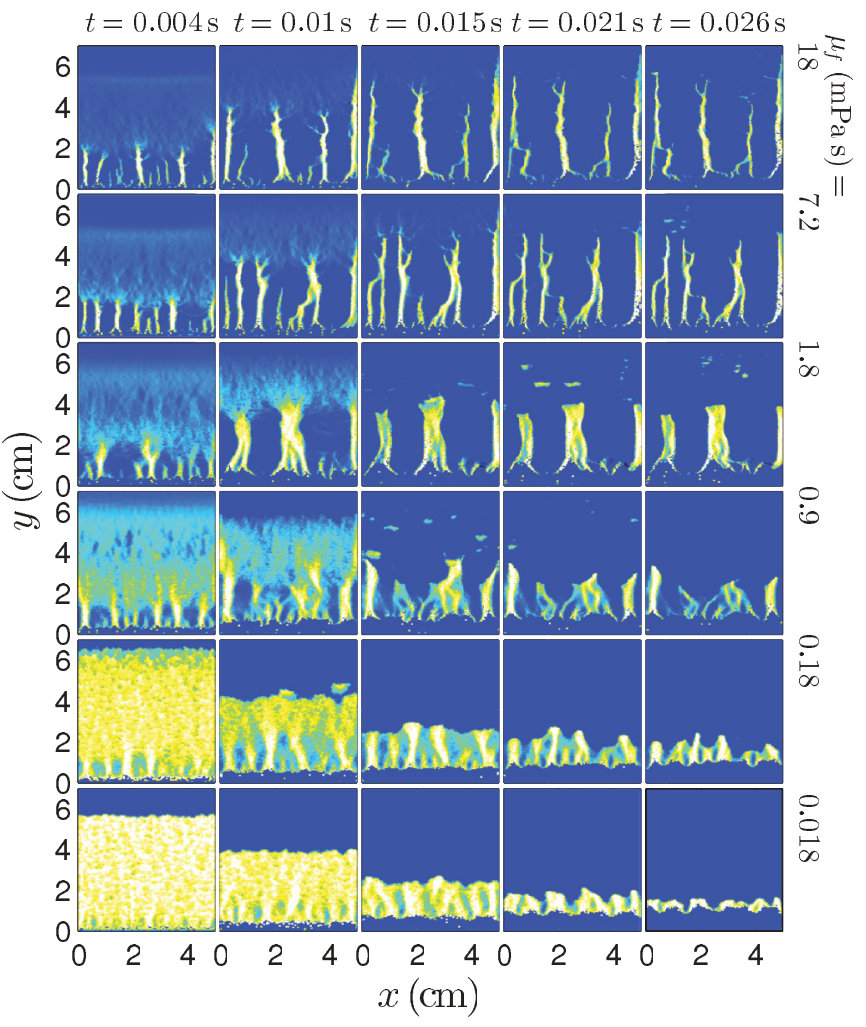

FIG. 7. (Color online) Snapshots of $u_{y}$, the $y$ component of the particle velocity, for decreasing gas viscosity (top to bottom) and as a function of time (left to right). High velocity appears yellow (brighter) in the snapshots. Here $u_{y}$ is normalized to one.

At the very start of the simulations the evolution of the system is controlled by two length scales. The first length scale is the length of the skin depth. The second length scale is the thickness of the compaction front at the gas-particle interface at the inlet. Before fractures appear the thickness of this compaction front is related to the growth of the particle free zone at the inlet. When the particle free zone at the inlet of the cell grows to a certain length of $Y_{t}$ in the $y$ direction from the inlet the displacement of the particles leads to a compaction

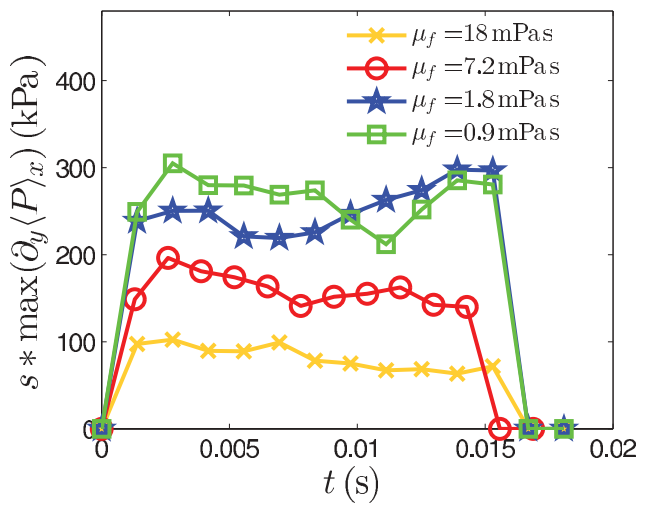

FIG. 9. (Color online) Close-up of Fig. 8(c) for the four highest viscosity values tested.

front with a minimal thickness of

$$
d_{c}=\frac{Y_{t} \rho_{s}^{(0)}}{\rho_{s}^{\max }-\rho_{s}^{(0)}},
$$

where $d_{c}$ depends on the initial solid volume $\rho_{s}^{(0)}$ fraction and the possible maximal volume fraction $\rho_{s}^{\max }$ of the grains. Before fractures appear $Y_{t}$ is equivalent to the position of the most advanced finger tip, which is plotted in Fig. 10. The figure shows that the particle free zone at the inlet progresses linearly in time for all viscosity values with constant velocity $\dot{Y}_{t}$ until complete particle compaction occurs at $t>0.01 \mathrm{~s}$. For the viscosity value of $\mu_{f}=0.18 \mathrm{mPa}$ distinct fingers and fractures are yet not formed. Nevertheless, the figure shows that the growing particle free zone at the pressure inlet already progresses linearly in time before fingers form. According to Eq. (6) this also implies a linear growth in time for the thickness of the compaction front. However, the pressure skin depth increases proportionally to the square root in time as shown in Eq. (5). At a certain transition time

$$
t_{f}=\frac{4 D}{\dot{d}_{c}^{2}}
$$

the thickness of the compaction front $d_{c}$ will therefore overtake the skin depth $s$.

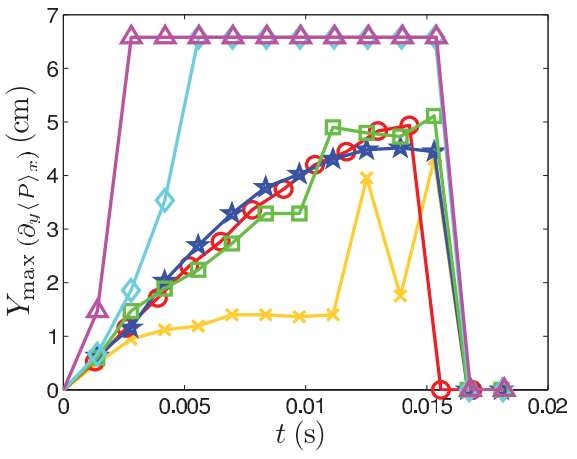

(a)

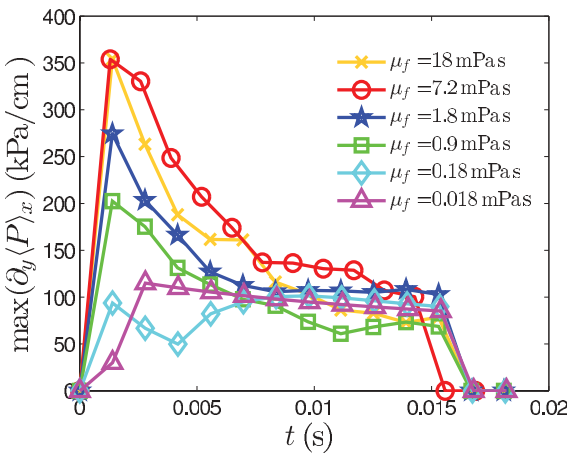

(b)

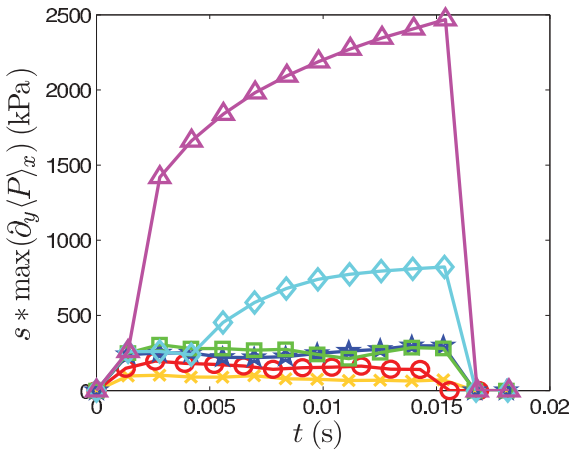

(c)

FIG. 8. (Color online) (a) The $y$ position of the maximum gradient in the $y$ direction of the average $\langle P\rangle_{x}$ of the pressure in the $x$ direction as a function in time. (b) Maximal gradient in the $y$ direction of the in $x$ direction averaged pressure: $\partial_{y}\langle P\rangle_{x}$ as a function in time. (c) This maximum derivative is rescaled by the skin depth $s=\sqrt{4 D t}$. The legends is consistent in all plots and is displayed only in (b). 


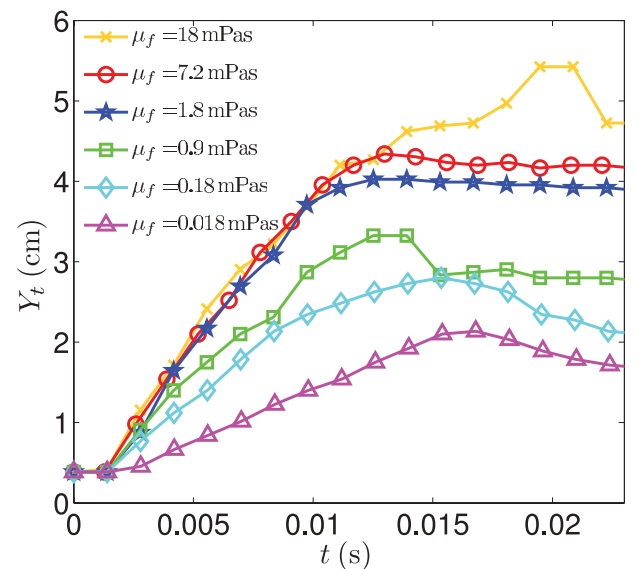

FIG. 10. (Color online) Position of the most advanced finger tip $Y_{t}$ as a function of time and for different viscosity values. The position of the most advanced finger was found by normalizing the particle density by its maximum value and averaging in the $x$ direction. If this averaged particle density drops to a value of 0.85 , approaching from the outlet side of the cell, we define the corresponding $y$ position $Y_{t}$ as the position of the most advanced finger tip.

At time $t_{f}$ we expect the transition from a body force to a surface force and the appearance of fractures instead of dispersed bubbles. For a direct comparison we visualized the transition time $t_{f}$ in Fig. 5 by a dashed (red) line. Above this line the thickness of the compaction front is larger than the skin depth. When the compaction front is ahead of the skin depth fractures were predicted in the previous discussion. The fractures in Figs. 2 and 5 emerge at the predicted time and thus demonstrate good agreement between the analytical prediction and the simulations.

After fingers emerge in the regime of high gas viscosity $\left(\mu_{f}>0.9 \mathrm{mPas}\right)$ the compaction front propagates at a constant speed through the cell. This is shown in Fig. 11(a), where the $y$ position of the maximum solid volume fraction averaged in the $x$ direction $\max \left(\left\langle\rho_{s}\right\rangle_{x}\right)$ is plotted in time. For a gas viscosity of $\mu_{f}<0.9 \mathrm{mPa}$ the compaction front manifests first at the outlet side of the cell, in good agreement with that previously discussed Fig. 3.

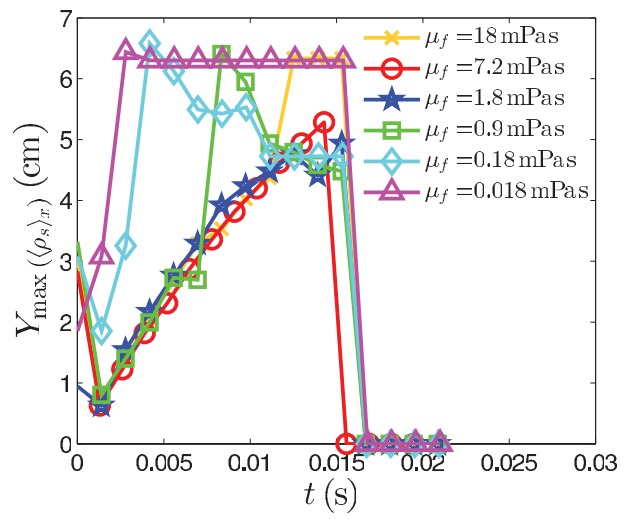

(a)
In Fig. 11(b) we plotted the thickness of the particle compaction front in time. Although the data are very noisy, it can be seen that the thickness of the compaction front for all tested viscosity values initially grows in time. The compaction front for a gas viscosity $\mu_{f}<0.9 \mathrm{mPa}$ s is located at the outlet of the cell and the thickness grows until complete compaction of the grains. The more interesting values are found for a gas viscosity of $\mu_{f}>1.8 \mathrm{mPa}$ because here the compaction front is located at the gas-particle interface and the thickness is measured during the fracturing of the granular packing is taking place. In this regime for the two highest viscosity values an initial increase of the compaction front thickness occurs. After a time of approximately $t=0.002 \mathrm{~s}$ the growth of the compaction front thickness slows down and appears to stay rather constant during the further propagation of the fractures until boundary effects start to play a role and the grains get completely compacted. This happens at $t \approx 0.01 \mathrm{~s}$ for the gas of highest viscosity and at $t \approx 0.007 \mathrm{~s}$ for the gas with a viscosity of $\mu_{f}=1.8 \mathrm{mPa}$.

Reducing the skin depth by increasing the viscosity has the same effect on the pressure evolution as increasing the system size instead. We can demonstrate this in the following discussion by nondimensionalizing Eq. (1). We have previously shown that when the gas viscosity is increased the pressure drop gets more and more localized along the interface between the gas and the grains. Thus a limit is approached continuously where only the outermost particles at the interface are accelerated by the pressure gradient. The presented simulations approach this limit of a pure surface force. As a consequence, the velocity of the finger tip in Fig. 10 reaches a maximum for gas viscosity values above $\mu_{f} \geqslant 0.9 \mathrm{mPa}$. By calculating the slope of the graphs in Fig. 10, this maximal characteristic velocity for the finger tips in the plots is measured to be around $U_{0}=470 \mathrm{~cm} / \mathrm{s}$ and stays constant for $t<0.01 \mathrm{~s}$. A further increase of the gas viscosity will not significantly increase the maximal velocity of the finger tips. In the limit of a pure surface force this maximal velocity is now primarily dependent on the injection pressure and on the properties of the granular phase. To nondimensionalize Eq. (1) we use this characteristic velocity $U_{0}$ to define a dimensionless velocity $u=U_{0} u^{\prime}$ and

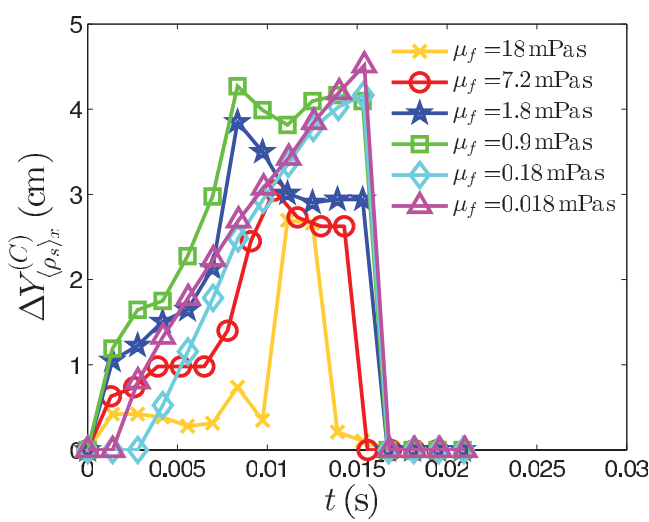

(b)

FIG. 11. (Color online) (a) The $y$ position of the maximum solid volume fraction $\max \left(\left\langle\rho_{s}\right\rangle_{x}\right)$ as a function of time. Before the position of the maximum is calculated the solid volume fraction in the cell was averaged along the $x$ direction. (b) A measure of the compaction front thickness $\Delta Y_{\left\langle\rho_{s}\right\rangle_{x}}^{(C)}$. It is the distance in the $y$ direction over which the $\left\langle\rho_{s}\right\rangle_{x}$ value lies above a threshold of 0.47 as a function of time. 
introduce $\kappa_{0}=a^{2} / 9 K$ as the characteristic magnitude of the permeability. To define further dimensionless variables we use the unit length $l$ as a characteristic length scale with $x=l x^{\prime}$ and $y=l y^{\prime}$. Scaling the system size by $l$ keeps the ratio between the $x$ and $y$ dimensions of the cell constant. When scaling the system size $l$ the rheology of the granular phase and the particle size remains unchanged. The atmospheric pressure $P_{0}$ is used as the characteristic pressure to define $P=P_{0} P^{\prime}$. From these quantities follows a characteristic time scale $\tau=l / U_{0}$ and $t=\tau t^{\prime}$ [22]. The resulting nondimensionalized equation is

$$
\begin{aligned}
& \phi\left[\frac{\partial P^{\prime}}{\partial t^{\prime}}+\mathbf{u}^{\prime} \cdot \nabla^{\prime} P^{\prime}\right] \\
& =\frac{1}{\operatorname{Pe}} \nabla^{\prime} \cdot\left[P^{\prime} \frac{\left(1-\rho_{s}\right)^{3}}{\rho_{s}^{2}} \nabla^{\prime} P^{\prime}\right]-P^{\prime} \nabla^{\prime} \cdot \mathbf{u}^{\prime} .
\end{aligned}
$$

In this equation the Péclet number was defined as

$$
\mathrm{Pe}=\frac{U_{0} \mu_{f} l}{P_{0} \kappa_{0}} .
$$

This analysis shows that alternatively changing the viscosity, the length scale, or the inverse permeability has the same effect on Eq. (8), which describes the pressure evolution. Increasing the gas viscosity is equivalent to using a larger system or reducing the permeability. In our simulations it leads to extremely time consuming calculations to decrease the particle size in order to reduce the permeability of the granular phase. Instead we chose to increase the viscosity to study the system dynamics at a small pressure skin depth. In contrast, in experiments it might be much easier to reduce the pressure skin depth by using smaller particles instead of increasing the cell size by a factor of 1000 .

\section{CONCLUSION}

The emerging structures sensitively depend on whether the particles are accelerated primarily by solid contacts or by the imposed pressure gradient. We could show that the evolving structures depend on a characteristic length scale that is given by the skin depth $s$. For a skin depth larger than the system size, large scale homogeneous motion and the formations of dispersed bubbles are the results. For a short skin depth compared to the system size a compaction front builds up that allows fracturing. Hence, controlling the time dependence of the injection pressure should in principle allow one to control the pressure response in the packing and transit from fracturing to diffusely compacting regimes. It should also in principle enable fracturing of a porous rock at adjustable distances from the inlet.

\section{ACKNOWLEDGMENTS}

We acknowledge the support from the Centre for Advanced Study at the Norwegian Academy of Science and Letters, the NFR, the University of Strasbourg, the CNRS-INSU, and the Alsace region through the REALISE network. We thank Joachim Mathiesen, Luiza Angelutha, Maud Schelstraete, Øistein Johnsen, Jan Ludvig Vinningland, Paul Meakin, Einat Aharonov, and Dion Weatherley for fruitful discussions.
[1] Ø. Johnsen, R. Toussaint, K. J. Måløy, and E. G. Flekkøy, Phys. Rev. E 74, 011301 (2006).

[2] X. Cheng, L. Xu, A. Patterson, H. M. Jaeger, and S. R. Nagel, Nat. Phys. 4, 234 (2008).

[3] Ø. Johnsen, C. Chevalier, A. Lindner, R. Toussaint, E. Clément, K. J. Måløy, E. G. Flekkøy, and J. Schmittbuhl, Phys. Rev. E 78, 051302 (2008).

[4] Ø. Johnsen, R. Toussaint, K. J. Måløy, E. G. Flekkøy, and J. Schmittbuhl, Phys. Rev. E 77, 011301 (2008).

[5] M. J. Niebling, R. Toussaint, E. G. Flekkøy, and K. J. Måløy, Rev. Cub. Fis. 29 (1E), 66 (2012).

[6] J. L. Vinningland, R. Toussaint, E. G. Flekkøy, and K. J. Måløy, in Complex Activity Report 2009, edited by E. G. Flekkøy and K. J. Måløy [http://www.complexphysics.org/Projects/PDF/ ComplexActivityReport2009.pdf], p. 108.

[7] A. Nermoen, C. Raufaste, S. D. de Villiers, E. Jettestuen, P. Meakin, and D. K. Dysthe, Phys. Rev. E 81, 061305 (2010).

[8] B. Sandnes, E. G. Flekkøy, H. A. Knudsen, K. J. Måløy, and H. See, Nat. Commun. 2, 288 (2011).

[9] R. Holtzman, M. L. Szulczewski, and R. Juanes, Phys. Rev. Lett. 108, 264504 (2012).

[10] H. Huang, F. Zhang, P. Callahan, and J. Ayoub, Phys. Rev. Lett. 108, 258001 (2012).

[11] S. D. de Villiers, A. Nermoen, B. Jamtveit, J. Mathiesen, P. Meakin, and S. C. Werner, Geophys. Res. Lett. 39, L13204 (2012).
[12] M. A. van der Hoef, M. van Sint Annaland, and J. A. M. Kuipers, Chem. Eng. Sci. 59, 5157 (2004).

[13] N. G. Deen, M. van Sint Annaland, M. A. van der Hoef, and J. A. M. Kuipers, Chem. Eng. Sci. 62, 28 (2007).

[14] N. G. Deen, M. van Sint Annaland, M. A. van der Hoef, and J. A. M. Kuipers, Prog. Comput. Fluid Dyn. 7, 152 (2007).

[15] M. A. van der Hoef, M. van Sint Annaland, N. G. Deen, and J. A. M. Kuipers, Annu. Rev. Fluid Mech. 40, 47 (2008).

[16] C. Zeilstra, J. G. Collignon, M. A. van der Hoef, and J. A. M. Kuipers, Powder Technol. 184, 166 (2008).

[17] T. Börzsönyi, R. E. Ecke, and J. N. McElwaine, Phys. Rev. Lett. 103, 178302 (2009).

[18] M. Schelstraete, Master's thesis, University of Strasbourg, 2009.

[19] M. J. Niebling, E. G. Flekkøy, K. J. Måløy, and R. Toussaint, Phys. Rev. E 82, 011301 (2010).

[20] M. J. Niebling, E. G. Flekkøy, K. J. Måløy, and R. Toussaint, Phys. Rev. E 82, 051302 (2010).

[21] J. L. Vinningland, R. Toussaint, M. Niebling, E. G. Flekkøy, and K. J. Måløy, Europhys. Lett. 204, 27 (2012).

[22] S. McNamara, E. G. Flekkøy, and K. J. Måløy, Phys. Rev. E 61, 4054 (2000).

[23] J. L. Vinningland, Ø. Johnsen, E. G. Flekkøy, R. Toussaint, and K. J. Måløy, Phys. Rev. Lett. 99, 048001 (2007).

[24] J. L. Vinningland, Ø. Johnsen, E. G. Flekkøy, R. Toussaint, and K. J. Måløy, Phys. Rev. E 76, 051306 (2007). 
[25] J. L. Vinningland, Ø. Johnsen, E. G. Flekkøy, R. Toussaint, and K. J. Måløy, Phys. Rev. E 81, 041308 (2010).

[26] D. V. Anghel, M. Strauss, S. McNamara, E. G. Flekkøy, and K. J. Måløy, Phys. Rev. E 74, 029906(E) (2006).

[27] P. C. Carman, Trans. Inst. Chem. Eng. 15, 150 (1937).

[28] E. G. Flekkøy, R. Delgado-Buscalioni, and P. V. Coveney, Phys. Rev. E 72, 026703 (2005).
[29] W. H. Press and W. T. Vetterling, Numerical Recipes (Cambridge University Press, Cambridge, 2002).

[30] See Supplemental Material at http://link.aps.org/supplemental/ 10.1103/PhysRevE.86.061315 for high-resolution movies of the particle density evolution during the compaction.

[31] E. Butkov, Mathematical Physics (Addison-Wesley, Reading, MA, 1968). 